Revue d'histoire de l'Amérique française

ZAS REVUE D.HISTOIRE DE L'AMÉRIQUE FRANÇAISE

\title{
Les Canadiens veulent conserver le régime seigneurial (1re partie)
}

\section{Marcel-Joseph}

Volume 7, numéro 1, juin 1953

URI : https://id.erudit.org/iderudit/301577ar

DOI : https://doi.org/10.7202/301577ar

Aller au sommaire du numéro

Éditeur(s)

Institut d'histoire de l'Amérique française

\section{ISSN}

0035-2357 (imprimé)

1492-1383 (numérique)

Découvrir la revue

\section{Citer cet article}

Marcel-Joseph (1953). Les Canadiens veulent conserver le régime seigneurial (1re partie). Revue d'histoire de l'Amérique française, 7(1), 45-63.

https://doi.org/10.7202/301577ar d'utilisation que vous pouvez consulter en ligne.

https://apropos.erudit.org/fr/usagers/politique-dutilisation/ 


\title{
LES CANADIENS VEULENT CONSERVER LE RÉGIME SEIGNEURIAL *
}

\author{
INTRODUCTION
}

Un grand historien de langue anglaise, William Bennett Munro, a répandu quelques idées fausses au sujet du régime seigneurial canadien. Il a prétendu, par exemple, que, dès son introduction dans le Bas-Canada, vers 1791, la façon anglaise de posséder les terres, appelée tenure en franc et commun soccage, conquit l'admiration des Canadiens, à cause de sa supériorité et de ses avantages.

Ce préjugé posé comme antécédent, l'historien, c'était fatal, a abouti à une conclusion absolument fausse, à savoir: que, vers 1820, ce n'étaient pas les seigneurs qui demandaient l'abolition de la tenure seigneuriale, mais bien les censitaires.

Quant à nous, nous soutenons que les Canadiens ne se laissèrent pas convaincre si facilement que cela, de la supériorité de la tenure anglaise sur la tenure dite française - quoique à ce moment elle n'existât plus en France -, que, même ẹn 1820, un certain nombre de gens des campagnes ne connaissaient rien de tout ce système nouveau, et que ceux qui en avaient la connaissance, tiraient de la comparaison des deux tenures une raison de plus de garder la leur. Ils demandaient, il est vrai, qu'on contraignît quelques seigneurs à observer les lois relatives à la tenure seigneuriale, mais rien de plus.

Nous affirmons aussi que ce sont des seigneurs plutôt que des censitaires, qui ont lancé les premières attaques contre le régime seigneurial, et que, sauf quelques exceptions, c'étaient les Britanniques et non les Canadiens, qui en demandaient alors l'abolition.

* Thèse présentée à la Faculté des Lettres de l'Université de Montréal pour l'obtention du grade de maître ès arts. 
Une enquête, menée par la Chambre de 1820 à 1824, a permis aux Canadiens d'exprimer leur opinion sur ce sujet. Grâce aux témoignages recueillis à cette occasion, nous saurons ce qu'ils en pensaient alors.

L'enquête nous renseignera aussi sur le grand malaise dont souffrait, en ces années, un peuple qui comptait exclusivement sur l'agriculture pour subsister: le manque de terre. Plus de terre pour les Canadiens dans la province de Québec! C'est un non-sens et pourtant telle est la réalité.

\section{CHAPITRE PREMIER}

\section{LES ARRERAGES DE LODS ET VENTES}

Première constatation de Milnes - Arrérages de lods et ventes - Mutation de tenure - Ses motifs - Loi pour le soulagement... - Opposition d'Osgoode - Sanction royale Application de la loi.

A l'été de 1799, Robert Shore-Milnes assumait la succession du gouvernement Prescott. Comme tout administrateur entrant en fonction, il étudia le milieu nouveau où s'exercerait à l'avenir son activite.

Un des traits économico-sociaux du Bas-Canada frappa le gouverneur: l'égalité des classes. Aristocrate-né, il cherchait vainement sur notre terre d'Amérique, quelque château digne du nom, quelque seigneur dont le train de vie pût lui remémorer les beaux équipages des nobles d'Europe. Il eut l'impression qu'il manquait une classe d'hommes en cette province: la classe supérieure, l'aristocratie. Il en existait bien une, mais de pacotille; elle était pauvre et sans influence sur la masse dont elle émergeait à peine. "Le seigneur a un pouvoir insignifiant, disait-il, et ne cherche guère à améliorer sa fortune par le commerce, ni à augmenter son influence ${ }^{1}$."

La cause de cette pauvreté? Il la voyait dans la tenure des terres, c'est-à-dire dans le régime seigneurial et les lois françaises

1. Milnes à Portland, 1er novembre 1800, A. G. Doughty et D. D. Mc Arthur, Documents relatifs à l'histoire constitutionnelle du Canada, 1791-1818 [ Cette compilation sera désormais citée sous le sigle DRHC/ (Ottawa], 1915), 253. 
qui le régissaient. Car d'une part la tenure seigneuriale ne fait pas du seigneur un grand propriétaire terrien comme is en existe, a cette époque, en Angleterre. Au contraire, ce sont les habitants qui "sont de fait les seuls propriétaires de presque toutes les terres cultivées du Bas-Canada". Ils "sont pratiquement indépendants du seigneur", lequel n'a que le revenu du moulin banal, les cens et rentes et les lods et ventes. D'autre part, comme l'étendue des propriétés sur lesquelles s'exercent ses privilèges "devient après quelques générations beaucoup moins considérable, par suite des subdivisions fréquentes de propriété auxquelles donnent lieu les lois françaises qui régissent l'héritage... dans bien des cas, le seigneur se voit dans une situation inférieure à celle du vassal"'. L'assertion était juste. "Les seigneurs, tant français qu'anglais, mènent une vie simple et ordinaire;" remarque l'Irlandais Weld qui était venu promener ses loisirs en Amérique quelques années auparavant; "car quoique les seigneuries, en général, soient vastes, très peu d'entre elles rapportent à leurs propriétaires un revenu très considérable"3. Je "ne crois pas, continue Milnes, que dans aucune partie du monde il y ait un pays où se trouve établie à ce point l'égalité de situation. Sauf dans les villes de Québec, de Montréal et de Trois-Rivières, on n'observe d'autre différence, en examinant la masse des Canadiens, que celle qui doit être attribuée aux avantages de localité, à un sol plus riche ou à une plus ou moins grande activitét."

Aux yeux de cet aristocrate, c'était un mal. Pour le duc de Portland, c'était "un mal certainement regrettable". Il aurait voulu y porter remède, mais comment? Il hasarda tout de même quelque suggestion pour encourager les seigneurs à travailler à "sortir de leur présente situation médiocre"5. Les grands de l'époque avaient l'habitude de se servir de l'aristocratie pour influencer la masse. Le nouveau lieutenant-gouverneur se serait senti plus rassuré, s'il avait pu compter sur un tel intermédiaire. "En cela il représentait la

2. Milnes a Portland, 1er novembre 1800, DRHC 1791-1818, 254.

3. I. Weld, Junior, Travels Through the States of North America, and the Provinces of Upper and Lower Canada, during the Years 1795, 1796, and 1797 (London, 1799), 230.

4. Milnes a Portland, 1er novembre 1800, DRHC, 1791-1818, 254.

5. Portland a Milnes, 6 janvier 1801, DRHC, 1791-1818, 259. 
véritable mentalité des ministres anglais de l'époque, lesquels, tous aristocrates, menaient rondement la lutte contre la démocratie"6. Bien convaincu de l'importance de la classe sociale à laquelle il appartenait et constatant par ailleurs que la tenure seigneuriale tenait les seigneurs canadiens dans la médiocrité, Milnes en vint à la conclusion que cette tenure était nuisible. Autant il importait de créer une aristocratie en cette province, autant il importait d'y faire disparaître la tenure seigneuriale.

Au cours de l'hiver, Milnes étudia à fond la situation de la Province. Faisant l'inventaire des ressources de celle-ci, il constata qu'une branche du revenu de la couronne était, depuis 1760, improductive: les lods et ventes de la censive de Sa Majesté?. L'expression lods et ventes désignait une taxe d'un douzième, due au seigneur sur toute vente ou échange d'immeuble effectuée par un censitaire. De ce montant, il était d'usage de rabattre un tiers, lorsque l'acheteur versait cet impôt dans les trois mois après la transaction $^{8}$; "ce droit avait constamment été en vigueur dans la province depuis son établissement,... c'était une taxe bien reconnue et établie, parfaitement acceptee par les habitants". Les seigneurs particuliers, cela va sans dire, avaient continué à percevoir cet impôt dans leur seigneurie, même après la conquête. Mais les administrations plutôt paternelles de Murray et de Carleton en avaient négligé la perception dans la censive de Sa Majesté, c'est-à-dire sur les terres où le roi lui-même était seigneur; lesquelles étaient "surtout situées dans les limites de la cité de Québec". Exiger le paiement de tous ces arrérages et gonfler ainsi le chiffre du revenu de la Province, magnifique début pour un gouverneur; rien de mieux pour montrer au duc de Portland toute la sollicitude que son subalterne apportait à sa

6. A. Desrosiers et C. Bertrand, Histoire du Canada (Montréal, 1933), 294. 269.

7. J. Sewell," "Rapport du procureur général", 4 avril 1801, DRHC, 1791-1818

8. V. Morin, Seigneurs et censitaires, castes disparues (Montréal, 1941), 91; J. Bouchette, Description Topographique de la province du Bas Canada, avec des remarques sur le Haut Canada, et sur les relations des deux provinces avec les EtatsUnis de l'Amérique (Londres, 1815), 12-13; J. Neilson, éd., Journal de la Chambre d'Assemblee du Bas-Canada, 9 (1801): 105. 269.

9. J. Sewell, "Rapport du procureur général", 4 avril 1801, DRHC, 1791-1818, 
tâche. La couronne en recevrait "une somme de cinq mille livres" sterling d'abord" "et ensuite une somme considérable chaque année $e^{11}$."

Tout en approfondissant la question, le lieutenant-gouverneur trouva ou d'autres l'aidèrent à trouver que la perception des arrérages de lods et ventes pouvait s'envisager sous un autre angle. Luimême nous le dit: "Lorsque je présentai à votre grâce mes premières remarques concernant ces choses [ la tenure seigneuriale], je ne prévoyais pas qu'il surgirait des circonstances grâce auxquelles ce qui restait du système féodal pourrait, avec le temps, être aboli du consentement mutuel du seigneur et de son tenancier; les renseignements supplémentaires que j'ai recueillis... [ sur ce sujet, en étudiant la possibilité de percevoir les arrérages d'impôts, j me portent à croire... qu'il est possible que le remède aux maux inhérents aux tenures actuelles provienne de cet acte de la législature". Ainsi, cette question des lods et ventes avait une double fin: percevoir une somme rondelette due à la couronne et, par là, provoquer du mécontentement et pousser les censitaires à demander d'eux-mêmes l'abolition de ces impôts et de la tenure seigneuriale qui en était la cause: "Il sautera aux yeux de Votre Grâce que, aussi longtemps que les lods et ventes dus à Sa Majesté ne sont pas réclamés, on ne saurait s'attendre à ce qu'aucune personne de la censive de Sa Majesté soit désireuse de voir ces redevances commuées". Leurs sentiments changeront lorsqu'ils seront obligés d'acquitter régulièrement leurs droits de mutation. Le désir leur viendra spontanément de s'exonérer de ces amendes et, pour cela, de changer la tenure de leurs terres ${ }^{12}$.

L'idée est lancée: essayons de détruire ces vestiges de la féodalité. Est-ce pour le plus grand bien du peuple? car enfin un homme d'Etat doit toujours avoir cette fin en vue. Les cultivateurs canadiens sont-ils malheureux? Là n'est pas la question, car, il le constatait lui-même quelques mois auparavant: "ces habitants, pouvant se procuı er par eux-mêmes d'une année à l'autre les choses nécessaires,

10. Ibid., 273.

11. Milnes à Portland, 16 avril 1801, DRHC, 1791-1818, 262.

12. Milnes à Portland, 10 juin 1801, DRHC, 1791-1818, 259 note 1. 
constituent la race la plus indépendante que je connaisse"13. Pourquoi alors ce désir de substituer la tenure anglaise à la française? "Par ce moyen la tenure féodale qui jusqu'ici a été un obstacle à l'acquisition, par les Anglais, de biens fonciers, sera écartée". Les terres, étant alors tenues en commun soccage, "les sujets anglais de Sa Majesté seront incités à devenir acquéreurs de vastes étendues que possèdent actuellement, par petites parties, les Canadiens. Et par là, Anglais et Canadiens se mêleront et, à la fin, une aristocratie des deux pourra être formée"14. Les vues des gouvernements sont parfois insondables! Qui, parmi les Canadiens du temps, aurait pu soupçonner que Milnes entretînt de telles pensées? Néanmoins, pour ne point inquiéter personne, le procureur général, Jonathan Sewell, étudia la question avec "des amis éprouvés du gouvernement... qui comptaient sur l'abolition graduelle de la tenure féodale"15. Le Lieutenant-Gouverneur, pour sa part, dit qu'il les encouragea "à mettre de l'avant le bill concernant les lods et ventes"16. Qu'est-ce à dire? Que nous nous trouvons en face d'un plan ourdi dans le secret par le Gouverneur et ses amis pour essayer de provoquer un mouvement contre la tenure seigneuriale.

Il s'agissait en effet de présenter devant la Chambre, à la prochaine session, un projet de loi visant à recouvrer les droits de mutation que les censitaires du roi avaient oublié de payer depuis la conquête. Ces droits étaient dus, tout le monde le savait. Mais on croyait qu'ils ne seraient jamais perçus. "Pour cette raison, dit Sewell, on a jugé opportun de réclamer les Lods et Ventes dus à Sa Majesté afin de convaincre les tenants [tenanciers] que la couronne n'abandonnerait pas une source de revenus susceptible de devenir extrêmement productive". On sait bien que cela ne plaira pas aux censitaires. Qu'à cela ne tienne! Ils n'auront qu'à le dire: c'est justement ça qu'on veut. Écoutons plutôt Sewell: "Et comme les terres tenues directement de Sa Majesté sont surtout situées dans les limites de la cité de Québec et appartiennent a des tenants [ tenanciers] anglais, qui sont pour la plupart des marchands tou

13. Milnes a Portland, 1er novembre 1800, DRHC, 1791-1818, 254.

14. Milnes à Portland, 10 juin 1801, DRHC, 1791-1818, 259 note 1. 269.

15. J. Sewell, "Rapport du procureur général", 4 avril 1801, DRHC, 1791-1818,

16. Milnes a Portland, 10 juin 1801, DRHC, 1791-1818, 259 note 1. 
opposés au paiement des Lods et Ventes... il était évident qu'une fois convaincus que les Lods et Ventes ne seraient pas abandonnés, ils demanderaient tous à grands cris une commutation, que par suite, le changement de leur mode de tenure en celui de franc et commun socage serait accordé à leur demande comme une faveur par le gouvernement, - car sans un changement de tenure il ne peut y avoir de commutation des Lods et Ventes."

On aura remarqué les mots "tenanciers anglais", "marchands tous opposés au paiement des Lods et Ventes". Pourquoi cela? Parce qu'ils considèrent les lods et ventes comme "une taxe... sur la valeur de toute amélioration du sol et, par conséquent comme un obstacle à tout motif d'activité". Cette taxe d'un douzième n'irritait pas trop les Canadiens français car, ordinairement, le bien paternel passait à l'un de enfants et, dans ce cas, l'héritier n'avait rien à payer au seigneur. Mais il en était autrement des marchands et des industriels anglais, une entreprise commerciale êtant susceptible de changer de mains plus souvent et d'accroître sa valeur plus rapidement qu'une propriété privée. Aussi les parrains du bill prévoyaientils les hauts cris.

"Cependant il ne saurait être question de percevoir tous les Lods et Ventes dus à Sa Majesté, car l'achat et la vente des lots de terre et des maisons de la basse ville de Québec... ont eu lieu tant de fois depuis la conquête, que sur toutes les terres et constructions il est dû à la couronne pour Lods et Ventes près de la valeur entière de celles-ci, sur un très grand nombre, plus de la valeur spécifique". On ne pouvait tout de même pas prendre à la gorge la population d'une ville entière. Or il était "incontestable que la perception des deux tiers de ce qui était dû causerait inévitablement la ruine de la ville de Québec". Considérant, d'autre part, "que les Lods et Ventes faisaient partie du revenu public avant la conquête et étaient affectés par Sa Majesté, dès l'année 1766, au paiement des dépenses nécessaires du gouvernement de Québec"... on crut bon de demander à l'Assemblée la permission de faire des remises plus considérables qu'il n'était d'usage. Toutefois, c'était surtout pour une autre raison "beaucoup plus importante" qu'on avait recours a l'autorité du Parlement provincial: on voulait "épargner à la couronne l'odieux de forcer la perception d'une taxe que l'on croyait abandonnée dans les seigneuries du roi." 
La pensée politique à la base de ce projet? La voici telle qu'élaborée par le coryphée du parti anglais Jonathan Sewell: "Quarante ans se sont écoulés depuis la conquête et le système féodal subsiste encore au détriment du gouvernement et de ses sujets" anglais. "Ce serait téméraire à l'extrême d'entreprendre de détruire ce système immédiatement, au milieu d'une population illettrée". Mieux vaut "se mettre à l'œuvre pour en obtenir l'abolition d'une manière graduelle". Mais pourquoi l'abolir? Pour donner aux Britanniques accès aux seigneuries, bien mieux situees, sur les bords du SaintLaurent, que ne l'étaient les Townships à l'arrière du territoire seigneurial. Car "L'Anglais déteste la tenure féodale et l'on en voit la meilleure preuve dans la situation actuelle de la colonie où ne se trouvent pas plus de cinquante tenanciers anglais dans toutes les seigneuries, bien que la population du pays atteigne le chiffre de deux cent mille âmes"17. Un document officiel du 27 janvier 1790 nous a laissé les noms des "Anciens Sujets de Sa Majesté" propriétaires ou co-propriétaires de fiefs et seigneuries: ils étaient au nombre de trente-neuf à cette époque ${ }^{18}$. Quelques autres Anglais ont vraisemblablement acquis des seigneuries dans la décade suivante, portant ainsi le nombre des Anglais seigneurs entre quarante et cinquante. Alors qu'à la même date, le nombre des "Nouveaux Sujets", c'est-à-dire des Canadiens, seigneurs ou co-seigneurs était de cent vingt-deux ${ }^{19}$. Toutefois, notait un voyageur, quoique plusieurs seigneuries soient actuellement en des mains britanniques, on ne rencontre que très peu d'agriculteurs anglais sur des terres soumises à cette tenure ${ }^{20}$. Sewell considérait comme une anomalie que, dans une colonie britannique, la région la mieux organisée et la plus prometteuse - la vallée commerciale du Saint-Laurent demeurât fermée aux natifs de la métropole: "La disparition des

17. Les citations précédentes sont extraites de: J. Sewell, "Rapport du procureur général", 4 avril 1801, DRHC, 1791-1818, 268-270.

18. P. Lymburner, "List of old Subjects proprietors of Fiefs and Seigniories in the province of Quebec", 27 janvier 1790, Archives publiques du Canada, BasCanada [ APC, Q] , 49: 189-191.

19. P. Lymburner, "List of new Subjects proprietors of Fiefs and Seigniories in the province of Quebec", 27 janvier 1790, APC, Q 49: 183-188.

20. I. Weld, Junior, Travels Through thd States of North America, and the Pro-

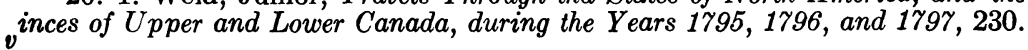


charges imposées par le régime féodal et le changement du mode de tenures en [ celui de] franc et commun soccage auront pour effet, en premier lieu, d'induire les gentilshommes anglais résidant au Canada, à faire l'achat de larges étendues de terre et d'induire aussi les petits propriétaires et les paysans anglais à devenir acquéreurs de lots plus petits dans les mêmes seigneuries, où ils refusent de s'établir à l'heure présente ${ }^{21}$."

Sewell escomptait encore beaucoup d'autres avantages: "Le changement... de tenure devra nécessairement opérer le mélange des Anglais et des Canadiens dans... les différentes seigneuries de la province; par suite, donner lieu à une confiance réciproque, introduire la langue anglaise et le mode anglais d'agriculture" et provoquer, en même temps, "l'assimilation des manières et des tendances". Pour cet homme, le Canada étrit non pas un pays indépendant, mais une colonie conquise et la Grande-Bretagne avait sur lui un droit de colonisation totale, suite du droit de conquête. C'est pourquoi il tenait ce langage. Mais ce n'est pas tout. "D'autre part, dans les endroits où les Canadiens sont mécontents, le gouvernement pourra de la sorte obtenir d'abord des renseignements et des avis à l'égard de leur conduite... bénéficier de la contrainte que leur imposeront un certain nombre d'Anglais résidant au milieu d'eux, puis finalement tirer parti d'une milice considérable et dévouée... ainsi que... d'un revenu annuel assuré et fixe substitué au profit incertain et inefficace des droits casuels de mutation."

Décidément, Sewell aurait eu raison de dire que "le bill était une mesure de politique éclairée" si tout cela s'était réalisé. Pourtant il n'en doutait pas. "Ces avantages paraissent assurés", disait-il. "Néanmoins il était compris que le résultat final en vue ne pouvait s'obtenir que graduellement;" pour y parvenir, "il était essentiel que l'exemple parte des seigneuries tenues directement de la couronne, afin de prouver par des faits" qu'il était possible et même avantageux de changer la tenure des terres; l'exemple, d'ailleurs, "serait beaucoup plus efficace si le changement était demandé comme une faveur à la couronne ${ }^{22}$." 268.

21. J. Sewell, "Rapport du procureur général", 4 avril 1801, DRHC, 1791-1818, 268-269. 
C'est le 26 janvier 1801 que l'occasion se présenta de jouer, à la Chambre, la séance si bien montée dans les coulisses. Un homme d'affaires de Montréal, James Mc Gill, se levant, demanda qu'on lât "le message de Son Excellence le Gouverneur-Général du 29 avril 1794, en tant qu'il se rapporte au revenu casuel, [ à ] celui des Domaines, et aux Lods et Ventes et quints dus à Sa Majesté." Ainsi fut fait"23. "Le Gouverneur, disait le texte en question, ne doute pas que la Chambre n'adopte des mesures pour soulager le Sujet par d'autres droits qui ne soulèveront pas d'objections, si la perception des Lods et Ventes, Droits de Quint etc. jusqu'à la limite légale doit être oppressive", ${ }^{24}$. Pourquoi ce message qu'on tirait soudain de la poussière des Journaux de la Chambre? Parce que tout acte à l'effet d'accorder des faveurs ou remises devait venir de la couronne ${ }^{25}$. De sa propre autorité, la Chambre ne pouvait s'immiscer dans cette question. Mais si le gouverneur l'y invitait, le débat était ouvert.

Cet appel de Lord Dorchester était-il donc resté sans réponse, la première fois qu'il avait été transmis à la Chambre? Le 14 décembre 1795, dit Sewell, il avait été résolu que la Chambre siégerait le 5 janvier pour étudier la question.

Et pour démontrer de quelle façon toutes les classes de la population interprétèrent le sens du message, il suffit d'indiquer que deux pétitions des habitants de Québec et de ses environs furent présentées à la Chambre, le 8 janvier 1796, et renvoyées au comité; que, dans l'une, les pétitionnaires demandaient "la remise de tous les droits seigneuriaux qu'ils devaient alors au domaine du roi" et dans l'autre "la remise de tous les Lods et Ventes dus à Sa Majesté dans la cité et les environs de Québec".

Le comité siégea à maintes reprises durant la session depuis le 5 janvier jusqu'au 23 mars, mais après avoir résolu "qu'il était expédient de faire la remise des... Lods et Ventes et quints dus à Sa Majesté..." les membres du comité constatè83.

23. J. Neilson, éd., Journal de la Chambre d'Assemblee du Bas-Canada. 9 (1801):

24. "Compte du revenu de la province depuis le commencement du nouveau régime jusqu'au 10 janvier 1794", 19 avril 1794, APC, Q 81-1: 193. 267.

25. "Protestation du juge en chef Osgoode", 27 février 1801, DRHC, 1791-1818, 
rent qu'ils ne pouvaient s'entendre... et, par suite, ils laissèrent le comité se démembrer et toute l'affaire fut abandonnée. Tout cela se passa sous les yeux mêmes de Lord Dorchester, sans qu'il ait jugé à propos de rien censurer ou désapprouver ou donné raison de croire qu'il en avait l'intention ${ }^{26}$.

C'est ainsi que la question des arrérages avait été remise dans le tiroir. Les censitaires de Québec la croyaient bien rendue aux oubliettes. Ils se trompaient. Quand quelqu'un oubliait, Sewell se souvenait. - Les citations précédentes montrent assez la part prépondérante qu'il eut dans cet épisode. - Cinq ans plus tard, à la Chambre d'Assemblée, la question était remise à l'ordre du jour. La Chambre se forma en comité plénier ${ }^{27}$. Les députés anglais, stylés par leur coryphée Sewell et remplis de "zèle pour l'abolition du système féodal" ${ }^{28}$, veillèrent à ce que le comité de 1801 fít mieux que celui de 1795. Au cours des débats, il y eut quelque velléité de suppression des lods et ventes dans les seigneuries du roi. Quelqu'un proposa de les remplacer par une taxe provinciale. Mais on lui fit remarquer que cette proposition était injuste: pour soulager le petit nombre de censitaires dépendant du roi, imposer une taxe a tous les habitants de la Province, lesquels payaient déjà ce même impôt à leur seigneur respectif ${ }^{29}$. Les députés durent renoncer à donner suite à la suggestion de Lord Dorchester les invitant à remplacer ces droits par d'autres.

Le rapport du comité de toute la Chambre, lu le 2 février 1801 par le président Berthelot ${ }^{30}$, déclarait que, si les lods et ventes étaient dus sur une seule mutation ou avaient "été réservés spécialement par contrat", il était "juste et expédient de les exiger et recevoir, en accordant le rabais accoutumé d'un tiers" à ceux qui les acquittaient dans le temps prescrit; mais que, dans tous les cas où ils étaient dus sur plus d'une mutation, il fallait accorder une certaine

26. J. Sewell, "Rapport du procureur général", 4 avril 1801, DRHC, 1791-1818, 93.

27. J. Neilson, éd., Journal de la Chambre d'Assemblée du Bas-Canada, 9 (1801): 270.

28. J. Sewell, "Rapport du procureur général", 4 avril 1801, DRHC, 1791-1818,

29. Milnes a Portland, 16 avril 1801, DRHC, 1791-1818, 262. 103.

30. J. Neilson, éd., Journal de la Chambre d'Assemblé du Bas-Canada, 9 (1801): 
remise, "suivant la nature et les circonstances de chaque cas particulier." La Chambre demandait enfin "que tous les droits de quint... actuellement dus" soient "exigés et reçus, avec le rabais accoutumé d'un tiers, s'ils sont payés" dans le temps prescrit ${ }^{31}$.

Cinq députés furent ensuite nommés pour préparer un bill conforme aux résolutions ci-dessus ${ }^{32}$. Ils rédigèrent un projet de loi intitulé: Acte pour le soulagement des personnes qui tiennent de $\mathrm{Sa}$ Majesté des terres ou propriétés immobilières en roture, sur lesquelles sont dus des droits de lods et ventes ou de mutation. Ce bill permettait au lieutenant-gouverneur de nommer cinq commissaires - la décision de trois d'entre eux étant finale - et un secrétaire pour percevoir les arrérages de lode et ventes et faire certaines remises jugées nécessaires ${ }^{33}$. "Aucun homme quel qu'il soit, notait Sewell, n'y est... dispensé du paiement des Lods et Ventes présentement dus sans le consentement de la couronne obtenu paı l'entremise des commissaires, sauf les personnes (et celles-là seulement) qui se sont distinguées dans la lutte pour la défense du gouvernement de Sa Majesté" ${ }^{34}$. L'article cinq, en effet, accordait remise complète aux personnes qui avaient participé à la défense de Québec en 1775 et dont les propriétés avaient été endommagées pendant le siège de la ville ${ }^{35}$.

Le bill franchit sans naufrage la Chambre basse. Contrairement à toute prévision, c'est au Conseil législatif qu'il reçut la plus forte opposition. Il subissait sa seconde lecture, lorsque le président, le juge en chef $\mathrm{Wm}$ Osgoode, crut trouver illégale l'initiative prise par l'Assemblée. Il y voyait un empiétement de celle-ci sur les pouvoirs de l'exécutif ${ }^{36}$. Ignorant le fin mot de la question, il s'opiniâtrait, seul contre tous, à vouloir préserver les droits de la couronne; alors que, comme le disait Sewell, le bill ne lui enlevait rien, mais lui permettait,

31. Ibid., 105.

32. Ibid., 109.

33. Ibid., 435; "Acte 41 Geo. 111, chap. 111", DRHC, 1791-1818, 263-266.

34. J. Sewell, "Rapport du procureur général", 4 avril 1801, DRHC, 1791-1818, 273.

35. "Acte 41 Geo. 111, chap, 111", DRHC, 1791-1818, 263-266.

36. "Protestation du juge en chef Osgoode", 27 février 1901, DRHC, 1791-1818, 266-267. 
au contraire, de percevoir une somme considérable ${ }^{37}$. Tout acte à l'effet d'accorder des remises doit venir de la couronne, disait Osgoode. Précisément, répondaient les autres conseillers, c'est sur une invitation expresse de Lord Dorchester, que l'Assemblée a entrepris l'étude de la question. L'Assemblée n'a pas agi lorsqu'elle y a été invitée, le 29 avril 1794, la question relève maintenant de la couronne seule, reprenait Osgoode ${ }^{38}$. L'invitation n'a jamais été révoquée; la Chambre était libre d'y répondre lorsqu'elle le jugerait opportun, soutenaient les autres membres du conseil: le lord-évêque de Québec, Hugh Finlay, François Baby, George Pownall, Henry Caldwell ${ }^{39}$. Le procureur général, interrogé, répondit: "A mon avis, le bill était une mesure... juste dans ses principes et présentée d'une manière constitutionnelle en vertu de l'autorité et avec l'approbation du gouvernement exécutif, et c'est pour cette raison que je l'ai appuyée" ${ }^{40}$. Malgré cette petite dissension, messieurs les conseillers virent à leurs intérêts: le bill sortit de leurs mains, amputé de la clause réclamant la perception des droits de quint ${ }^{41}$.

Afin de pouvoir procéder en connaissance de cause au recouvrement des arrérages d'impôts, l'Assemblée jugea nécessaire de faire confectionner ce qu'on appelait alors le papier terrier et censier, ou plus souvent le papier terrier, ou même le terrier. Elle en fit la demande au lieutenant-gouverneur, le 2 février $1801^{42}$. Le papier terrier était en quelque sorte un cadastre de la seigneurie, doublé d'un inventaire minutieux de chaque ferme située sur les terres du seigneur. Quand ce dernier annonçait qu'il ferait le terrier, chaque censitaire devait passer chez le notaire avec son titre de concession, c'est-à-dire l'écrit indiquant l'es redevances dont sa terre était 273. 266-267.

37. J. Sewell, "Rapport du procureur général", 4 avril 1801, DRHC, 1791-1818,

38. "Protestation du juge en chef Osgoode", 27 février 1801, DRHC, 1791-1818,

39. "Réponse à la protestation du juge en chef Wm Osgoode", 27 mars 1801, DRHC, 1791-1818, 267 note 1. $1818,268$.

40. J. Sewell, "Rapport du procureur général", 4 avril 1801, DRHC, 1791-

41. "Acte 41 Geo. 111, chap. 111", DRHC, 1791-1818, 263-266; J. Neilson éd., Journal de la Chambre d'Assemblée du Bas-Canada, 9 (1801): 105.

42. Ibid., 109-111. 
chargée, pour employer l'expression du temps. L'homme de loi transcrivait ce titre de concession et remett.iit au censitaire la copie qu'il venait de signer: c'était son "titre nouvel". Après quoi l'habitant énumérait en détril tous les biens immeubles et meubles qu'il possédait, pendant que le notaire en prenait note. Quand tous les cultivateurs avaient fait dresser leur inventaire, on rédigeait, à l'aide de tous ces écrits, le papier terrier que Milnes définissait: "un registre contenant l'état des revenus ou un tableau des terres tenues d'un seigneur en fief ou en roture, ainsi que la description de ces terres, des rentes, redevances, impôts, services et droits seigneuriaux auxquels sont assujettis les vassaux". Donnant suite à la demande de l'Assemblée, l'́ lieutenant-gouverneur forma un comité composé de tout le conseil exécutif pour décider des mesures à adopter pour la confection du terrier ${ }^{43}$. Le comité, ayant étudié la question, rédigea son rapport. Osgoode, qui maintenait toujours ses positions, ajouta, sous la signature du président du comité, une phrase de protestation contre ce nouvel empiétement de la Chambre ${ }^{44}$. Une controverse s'ensuivit. Le conseil s'indigna et déclara la protestation ${ }^{45} \mathrm{du}$ juge en chef "irrégulière dans sa forme et sa substance" et en demanda la suppression. On recourut ensuite au verdict du procureur général lequel, cela va sans dire, donna tort au juge en chef $^{46}$.

Malgré les protestations d'Osgoode, le 8 avril 1801, le lieutenantgouverneur Milnes annonçait que l'Acte pour le soulagement des personnes qui tiennent de Sa Majesté, en roture, des terres ou immeubles sur lesquels des lods et ventes sont dus, était approuvét ${ }^{47}$. Le duc de Portland, mis au courant des faits, approuva l'Acte passé dans la 41e du règne de Georges III, chapitre III et tout ce que l'Assemblée avait fait ${ }^{48}$.

43. Milnes à Portland, 15 mai 1801, DRHC, 1791-1818, 274-275. 276.

44. "Protestation du juge en chef Osgoode", 1er mai 1801, DRHC, 1791-1818, 227.

45. "Minute du Conseil", 5 mai 1801, 8 mai 1801, APC, Q 86-2: 221, Q 87-2:

46. "Rapport du comité du Conseil exécutif", 7 mai 1801, J. Sewell, "Rapport du procureur général", 15 mai 1801, DRHC, 1791-1818, 277-280. 435.

47. J. Neilson, éd., Journal de la Chambre d'Assemblee du Bas-Canada, 9 (1801):

48. Portland à Milnes, 13 juillet 1801, DRHC, 1791-1818, 284. 
Faut-il voir, dans l'opposition du juge en chef et président du conseil exécutif $\mathrm{Wm}$ Osgoode, une preuve que l'affaire ne venait pas du parti anglais? Non pas. Car Osgoode, quoique Anglais, n'était pas du nombre des "amis éprouvés du gouvernement" dont parlait Sewell. C'était un homme froid et indépendant. Comme membre de l'exécutif, il avait eu des passes d'arme avec Lord Dorchester, Prescott et Milnes. Au moment même où ces discussions avaient lieu, la question de son remplacement était à l'étude ${ }^{49}$. Milnes lui-même avouait, quoique indirectement, qu'il était brouillé avec son subordonne $e^{50}$. Il ne faut donc pas s'étonner de voir celui-ci dans l'opposition. Ses protestations ne changent rien au fait que le groupe anglais entendait partir de la réclamation des lods et ventes, pour déclancher une campagne d'opinion contre la tenure seigneuriale qu'ils abhorraient.

Le 13 mai 1801, les commissaires nommés pour appliquer la loi votée à la dernière session se réunissaient à Québec $^{51}$. Étaient présents: le président Thomas Dunn, conseiller législatif et exécutif et ancien président de la commission pour l'administration et l'amélioration des biens des Jésuites ${ }^{52}$, François Baby, Gabriel-Elzéar Taschereau et Robert Lester ${ }^{53}$, ainsi que le secrétaire du comité Olivier Perrault ${ }^{54}$. Ce jour-là, notait ce dernier, les commissaires résolurent qu'un avertissement soit inséré en les deux langues et par trois fois dans la Gazette de Québec, donnant avis public qu'ils tiendront leurs séances dans une chambre de l'évêché, tous les mardis et vendredis de chaque semaine (les fêtes exceptées) à commencer le 15 mai, "à l'effet de recevoir de toutes personnes intéressées leurs

49. DRHC, 1891-1818, 34 note 1 .

50. Milnes à Portland, 12 juin 1801, DRHC, 1791-1818, 281.

51. Journal des procédés des Commissaires nommés et appointés par son Excellence le Lieutenant Gouverneur Robert Shore Milnes Ecuyer, en vertu d'un Acte du Parlement Provincial du Bas Canada, intitulé "Acte pour le soulagement des personnes qui tiennent des Terres ou Immeubles de sa Majesté, en roture, sur lesquels des Lots et Ventes sont dus [ Délibérations des Commissaires de 1801. E, no 32], 1.

52. Milnes à Portland, 7 août 1801, APC, Q 87-1: 76.

53. Délibérations des Commissaires de 1801. E, no 32, 2.

54. I. Caron, La Colonisation de la province de Québec. Les Cantons de l'Est 1791-1815, (Québec, 1927), 159 note 2. 
prétensions respectives, pour un abandon ou remise sur aucun droit de Lods et Ventes, qui à la passation du susdit acte pourroit être du par telles personnes; afin que les dits Commissaires puissent faire droit sur icelles, suivant et au désir de l'intention du susdit acte $^{55}$."

Le mois suivant, le comité était completé par l'arrivée de l'Honorable Joseph de Longueuil, lequel prêta le serment d'office le 5 juin $1801^{56}$. Le comité siégea jusqu'au 4 août $1802^{57}$. Aux jours fixés, les commissaires recevaient les censitaires de Sa Majesté. Ceux-ci exposaient, avec documents à l'appui, comment leur propriété leur était parvenue et combien de fois elle avait changé de mains depuis la conquête. Le secrétaire transcrivait leurs actes de vente et d'achat dans un registre ${ }^{58}$ et, à l'aide de ces contrats, les commissaires supputaient le montant des lods et ventes accumulés depuis 1760. Les Québécois mettaient alors une humilité remarquable à étaler leur pauvreté devant les agents du fisc! Ils obtenaient des réductions importantes. Quelques-uns obtenaient même la remise complète de leurs dus ${ }^{59}$.

Du 6 janvier 1799 au 5 janvier 1800, aucun droit de lods et ventes n'avait été payé à lá couronne ${ }^{60}$. De cette dernière date au 5 janvier 1801, seul l'honorable Thomas Dunn, qui voyait sans doute venir l'heure du règlement des comptes, s'était mis en règle en versant au receveur général la somme de 25 livres $2 \mathrm{~s} 6 \mathrm{~d}$ "sur l'acquisition d'une maison dans le Sault-au-Matelot, à Québec" ${ }^{61}$. C'était maigre, comparé au montant réalisé en 1759 , dernière année du gouverneur français, alors qu'il s'était élevé à neuf cents livres sterling environ $^{62}$. La loi de 1801 changea le cours des choses. Le receveur

55. Délibérations des Commissaires de 1801. E, no 32, 1.

56. Ibid., 5 .

57. I. Caron, La Colonisation de la province de Québec. Les Cantons de l'Est 17911815,159 note 2 .

58. Terrains du Roi et observations. B.

59. Délibérations des Commissaires de 1801. E, no 32. 181.

60. J. Neilson, éd., Journal de la Chambre d'Assemblée du Bas-Canada, 8 (1800): 247.

61. J. Neilson, éd., Journal de la Chambre d'Assemblee du Bas-Canada, 9 (1801):

62. Milnes à Portland, 16 avril 1801, DRHC, 1791-1818, 262. 
général reçut, entre le 6 janvier 1801 et le 5 janvier 1803, des lods et ventes pour un total de 5525 livres $7 \mathrm{~s} 61 / 2 \mathrm{~d}$ dus sur 293 propriétés, tant en arrérages qu'en ímpôts sur des mutations récentes ${ }^{63}$. Les prévisions de Sewell étaient réalissées en ce qui concernait le point de vue pécuniaire; au delà de cinq mille livres encaissées en un an, alors qu'on n'avait perçu que 1351 livres $9 \mathrm{~s} 51 / 4 \mathrm{~d}$ dans les quarante années précédentes ${ }^{64}$. Le lieutenant-gouverneur, peut-être en récompense de son zèle, probablèment à cause de l'augmentation des revenus, - Milnes lui-même fait le rapprochement des deux idées dans une lettre - verrait dorénavant son traitement augmenté et porté à 1500 livres, ce que, à l'en croire, il méritait bien ${ }^{65}$.

Restait la fin éloignée, celle que le parti anglais semblait avoir tant à cœur, celle dont Milnes avait écrit à Portland en ces termes: "Il est probable qu'à la prochaine session un bill sera présenté à l'effet de commuer les Lods et Ventes en [ la ] censive de Sa Majesté ce qui n'aurait jamais été effectué lorsqu'on espérait qu'ils ne seraient pas réclamés; et l'on a raison de croire qu'une semblable commutation s'effectuera dans les autres seigneuries" ${ }^{\prime 66}$. Le 4 octobre 1802, au cours d'une longue, "secrète et confidentielle" a pologie de son administration, Milnes revenait sur la question d'un changement de tenure: c'était, avec un bill qu'il préparait pour l'amélioration de la milice, l'une des deux plus importantes questions qu'il avait à étudier ${ }^{67}$. C'est assez dire l'importance qu'il y attachait. Car, en ces années où le Premier Consul faisait trembler la fière Albion, le Canada n'avait-il pas raison de craindre une invasion américaine au premier signal de guerre entre la France et l'Angleterre? La loi de milice fut effectivement votée à la session de $1803^{68}$ et, en mai, les deux puissances européennes étaient aux prises. La guerre occupa dès lors les esprits. De loi de mutation, point. Le silence se fit dès lors sur la

63. J. Neilson, éd., Journaux de la Chambre d'Assemblée du Bas-Canada, 10 (1802): $157 ; 11$ (1803): $51-61 ; 12$ (1804): 115-117. 273.

64. J. Sewell, "Rapport du procureur général", 4 avril 1801, $D R H C, 1791-1818$,

65. Milnes à Hobart, 4 octobre 1802, APC, Q 89: 126, 129.

66. Milnes a Portland, 16 avril 1801, DRHC, 1791-1818, 262.

67. Milnes à Hobart, 4 octobre 1802, APC, Q 89: 125.

68. A. Desrosiers et C. Bertrand, Histoire du Canada, 295. 
question. Le lieutenant-gouverneur lui-même n'en parla plus dans ses dépêches.

Il continua tout de même à avoir l'œil aux revenus de l'a Province. Mais toujours le receveur général s'objectait à ce qu'il exerçât un contrôle sur les droits de mutation. Henry Caldwell ne consentait même pas à donner de reçu ni de formule d'acquittement à ceux qui réglaient avec lui, en sorte qu'on ne pouvait vérifier cette partie de ses comptes ${ }^{69}$. Il accordait des sursis et faisait des remises ${ }^{70}$, ce qui revenait de droit au gouvelneur ${ }^{71}$. Ayant grand besoin d'argent pour achever de payer la seigneurie de Lauzon et les autres propriétés du général Murray dont il était propriétaire depuis 1801 ainsi que les seigneuries de St-Étienne et de Gaspé qu'il venait d'acheter ${ }^{72}$, il ne tenait pas du tout à être surveillé. En le suivant de piès, Milnes s'aperçut qu'il faisait "des erreurs" "73. L'affaire fut soumise au conseil exécutif, lequel fut d'avis que le receveur général n'était pas assez suivi dans la perception des lods et ventes. Il ne voulut pas enquêter sur le passé, mais décida de prendre des précautions pour l'avenirir ${ }^{74}$. Il demanda que désormais le censitaire passât d'abord chez l'inspecteur du domaine du roi pour faire évaluer le montant dû à la couronne, qu'il allât ensuite en demander la remise du tiers au Gouverneur et réglât dans les trois mois avec le receveur général ${ }^{75}$. Caldwell avait sûrement de grands intérêts à protéger puisqu'il porta devant Lord Hobart une cause d'ailleurs perdue d'avance ${ }^{76}$.

Depuis 1802 Milnes demandait à repasser l'Océan ${ }^{77}$. Le cinq août 1805, il s'embarqua pour l'Angleterre. Quatre ans après sa

69. Milnes à Hobart, 30 juin 1803, APC, Q 92: 85-86.

70. Ibid., 94-95.

71. Ibid., 88. 357-359.

72. J.-E. Roy, Histoire de la seigneurie de Lauzon, (5 vol., 1897-1904), 3:

73. Milnes à Hobart, 30 juin 1803, APC, Q 92: 85.

74. Ibid., 93.

75. "Rapport d'un comité de tout le conseil", 14 janvier 1803, APC, Q 92: 103; "Ordre concernant le paiement des droits de mutation", 10 août 1803, APC, Q 92: 349 .

76. Henry Caldwell à Hobart, 26 avril 1804, APC, Q 96: 77.

77. Milnes à Hobart, 4 octobre 1802, APC, Q 89: 129. 
passation, l'Acte pour le soulagement... n'avait encore déclanché aucune demande de mutation. Nulle loi n'était venue changer l'état des choses dans la censive du roi et plusieurs aristocrates devaient passer au Château St-Louis avant que s'effectuât la mutation de tenure rêvée par Sewell et Milnes.

Frère MARCEL-JosePH, S.G.

(à suivre)

Messire Pieire Mennard (1799-1792) Voir, vol. VI, no 4: 560-563.

Nous regrettons, faute d'espace, d'être obligé de remettre à notre prochaine livraison, des notes intéressantes qui viennent compléter celles que nous avait déjà fournies Monsieur Jean-Jacques Lefebvre, sur Messire Pierre Mennard (1739-1792). (NDLR) 